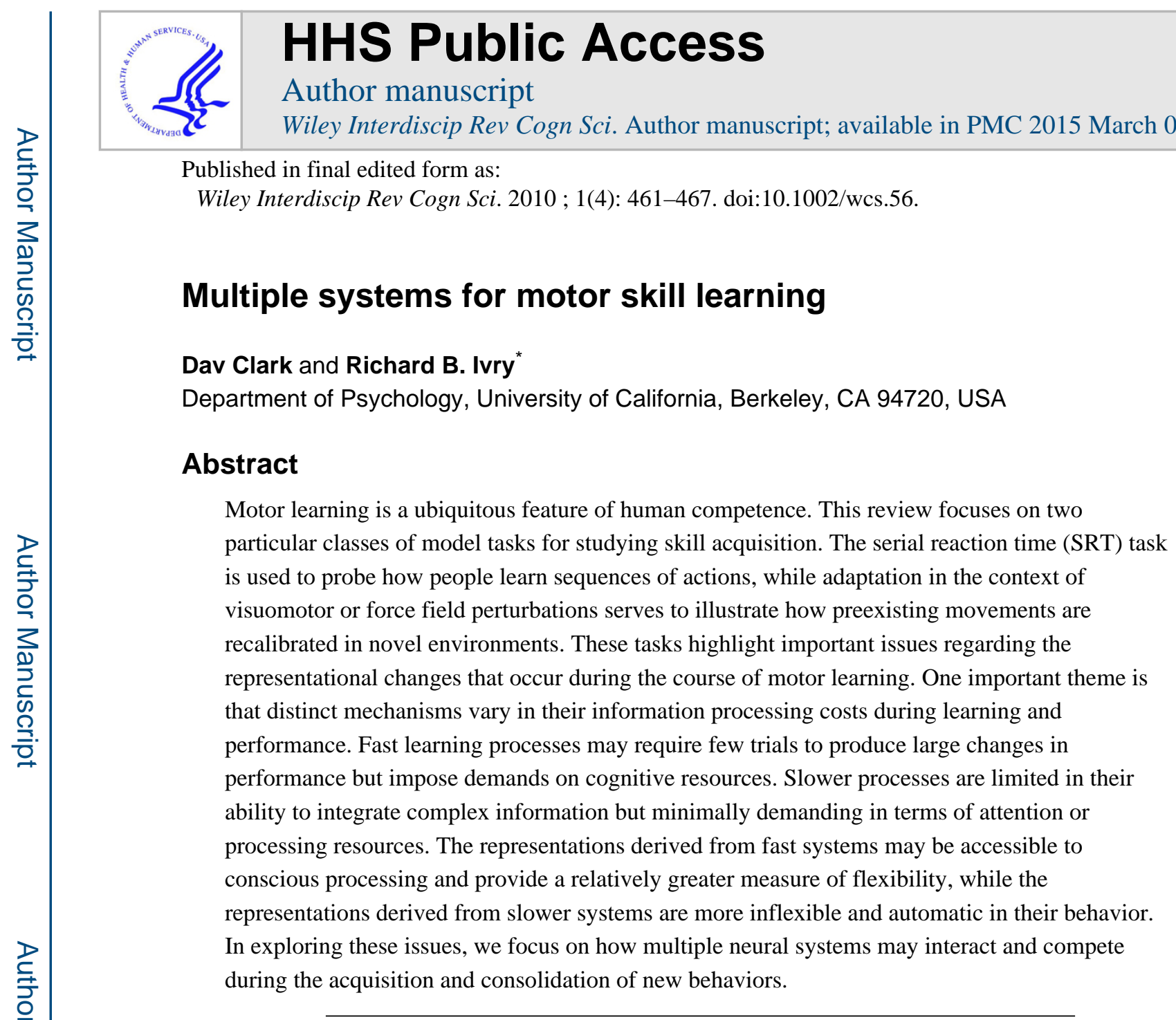

Motor skill can be loosely defined as an action with some metric of 'success' or 'quality.' With practice, skills are characterized by increased success with respect to externally defined criteria and increased quality and consistency of the constituent movements. Unlike habits or reflexes, skills involve intentional acts that are subjectively experienced. Abilities, such as sports or dance, require extensive practice and involve coordination of multiple limbs and even interactions between individuals. More prosaic examples such as the skilled use of eating utensils or the operation of a complex control panel involve simpler movements but still can exhibit vast differences in performance between the novice and expert.

Skilled performance reflects the interplay of a variety of factors. Factors such as biomechanics, postural control, and reflexive constraints are related directly to the neuromuscular system. More cognitive factors such as working memory, perception, and categorization are not typically thought of as relevant for understanding variation in motor performance, yet extensive lines of research suggest that these may impose fundamental constraints on skill acquisition. The interaction of multiple factors creates the potential for

(C) 2010 John Wiley \& Sons, Ltd.

“Correspondence to: ivry@ berkeley.edu. 
facilitation or interference between processes engaged during learning and performance. ${ }^{1,2}$ For example, instructional manipulations may influence on-line performance in such a way that some learning systems are inhibited. ${ }^{3}$ Below, we describe a core set of processes for motor skill and relate these to more general principles of learning.

\section{CHARACTERIZING BEHAVIOR}

Early studies on skill acquisition identified lawful relations between performance and properties of the task. The Hick-Hyman law describes how reaction time increases with information uncertainty while Fitts' law describes trade-offs between speed, accuracy, and movement amplitude. These laws break down with extensive practice, ${ }^{4}$ implying that motor performance is dependent on acquired knowledge about the goal structure of the task. Newell and Rosenbloom ${ }^{5}$ examined performance improvements over a large class of tasks, ranging from simple reaction time tasks to complex skills such as cigar rolling. Across these diverse domains, performance time decreases linearly with the amount of practice when plotted in log-log coordinates. This decrease is attributed to an underlying 'chunking' process through which components of the task are combined into functional units. A wellstudied example of chunking is typing. The performance of skilled typists suggests that they ultimately acquire chunks at multiple levels of a hierarchical structure such as that associated with representations of letter combinations, words, or phrases. ${ }^{6}$

Typing captures two important features of skill acquisition: the concatenation of cooccurring actions into integrated representations and the improvement in fluidity of the constituent parts. For example, a skilled typist will be faster in producing common sequences of key presses even when recalibrating her actions to conform to the novel configurations required by a specialized, ergonomic keyboard. The time course of behavioral improvements in performance, as well as the subsequent influence of this learning on other related tasks, has provided a rich data set to study motor learning.

\section{Sequence Learning}

A model task for studying sequence learning is the serial reaction time (SRT) task (Figure 1). In a common version of this task, a series of stimuli are presented in succession and participants are required to press the corresponding response key to each stimulus-much like a simplified version of ordinary typing. The stimuli may repeat in an exact or probabilistic structure, or they may be selected randomly. The reduction in reaction time to predictable stimuli provides a behavioral measure of sequence learning. ${ }^{7}$ The flexibility of a skill can be assessed by examining performance in a novel context. With transfer designs, one can ask how well training with one effector generalizes to other effectors or changes in the workspace. Training on the SRT task with finger movements of one hand shows considerable transfer when the responses are made with arm movements, finger movements with the other hand, or even verbal responses. ${ }^{8,9}$ Interestingly, during intermanual transfer, benefits are seen if the same stimulus sequence is unchanged or mirror-reversed. In the former, the pattern of finger movements is retained in exocentric coordinates; in the latter, the response sequence is the same in egocentric (finger) coordinates. 
The degree of transfer varies with the level of practice. Whereas intermanual transfer (in either coordinate space) is evident after $1 \mathrm{~h}$ of practice, little to no intermanual transfer is found after 5 weeks of training. ${ }^{10}$ This observation might suggest that extended practice may tie a skill to a particular mode of execution. The absence of transfer suggests that participants failed to develop or maintain more abstract levels of representation. However, this example fails to capture an important feature of skill; the jazz virtuoso continuously improvises new riffs on an old standard. It may be that extended practice produces different effects within the motor hierarchy. At some levels, consolidation may limit flexibility, ensuring that the movement is produced with minimal variation to ensure stability. At other levels, consolidation may create chunks that can be flexibly recombined and varied to explore new patterns.

Variation in generalization may also reflect the operation of multiple learning mechanisms that operate at different timescales. Early in training, fast learning processes may result in the development of abstract representations of the task domain that readily generalize across effectors and output spaces. With extended practice, slower processes that produce changes in an effector-specific manner come to dominate performance. Whereas faster systems may be disrupted by unrelated task requirements such as the presence of an irrelevant secondary task, slower systems may be more automatized. An interesting question for future research would be to explore the consequences of an extended training program that encouraged exploration of the workspace or perhaps the development of more abstract strategies rather than more traditional measures that focus on establishing stability within a limited context.

The SRT task has also been employed to explore constraints on learning. One contrast has been between sequences that include unique, local associations (e.g., the transition 1-3-2 in the sequence 1-3-2-3-2) and those in which local associations are ambiguous (e.g., 1-3-2-3-1-2). In the former, learning occurs even under divided attention manipulations. In contrast, learning under such conditions is minimal in the latter. ${ }^{8}$ Ambiguous sequences may require the integration of second-order contingencies. In terms of a hierarchical organization, higher order 'chunks' are necessary to resolve ambiguity. Such processes may be dependent on some form of awareness. However, explicit sequence awareness during training neither improves nor degrades subsequent performance with divided attention suggesting multiple, independent learning systems. ${ }^{11,12}$

Building on this idea, an alternative to the implicit/explicit characterization can be developed by considering the kinds of information that are available to different learning systems. ${ }^{12}$ The formation of explicit memories is known to depend on the medial temporal lobes (MTLs). However, more recent descriptions have emphasized that this region is essential for the formation of contextual or multidimensional associations. While this functional characterization has been widely recognized in the domain of episodic memory, ${ }^{13}$ it also appears useful for making sense of the functional imaging literature on sequence learning. ${ }^{14}$ Specifically, even under implicit learning conditions, we can observe MTLdependent learning if expectancies require integrating information across multiple inputs. ${ }^{12}$ When such integration is not required (or might even result in interference), learning may be limited to modality/effector-specific cortical regions or subcortical structures such as the basal ganglia or cerebellum. 


\section{Adaptation}

Parallel learning systems that operate on multiple timescales have also been hypothesized to account for motor learning observed in adaptation tasks. In visuomotor adaptation, participants reach to a target location but the input-output mapping is altered; for example, visual feedback of hand position may be rotated in a fixed relationship (Figure 2(a,b)). In other tasks, the environmental dynamics are altered such that the arm experiences perturbing forces over the course of the movement. Adaptation functions typically have a negative exponential shape, reminiscent of what one sees in the learning functions described by Newell and Rosenbloom. ${ }^{5}$ While one may interpret this function as reflecting the effect of diminishing returns, this behavioral profile can be accurately modeled as the result of multiple systems learning in parallel. ${ }^{15} \mathrm{Here}$, a fast process yields rapid learning but also rapid decay, even when the perturbation is maintained. A slower process requires more trials for a given amount of adaptation but is also relatively stable (i.e., the adaptation decays slowly). It remains to be seen if these different learning processes have similar characteristics to those observed in studies of sequence learning. For example, does a fast system for adaptation support relatively abstract representations that lead to generalization?

As with sequence learning, the issue of awareness has also been examined in the context of adaptation tasks. Participants will generally perceive a perturbation above some threshold and will generally not perceive a perturbation if it is introduced gradually. Thus, manipulating awareness usually requires a change in the magnitude of the perturbation to be learned. Nonetheless, there are ways to tease apart the contributions of explicit and implicit learning mechanisms. In one study, ${ }^{16}$ a $45^{\circ}$ visuomotor rotation was introduced and participants were told to strategically compensate for this by aiming at a neigh-boring target positioned $45^{\circ}$ opposite the direction of the rotation. As expected, the conscious strategy allowed participants to 'adapt' correctly on the very first trial. Surprisingly, though, accuracy deteriorated over time with participants producing larger rotations than required.

This paradox can be resolved by proposing distinct learning processes that operate on different sources of information. One system with access to strategic instructions may generate a motor plan that requires aiming to a strategic location rotated $45^{\circ}$ from the actual target. Another system may lack information about the actual target and instead calibrate the movement based on the difference between the predicted and perceived endpoints of the movement (Figure 2(c)). When these two systems are combined, one based on a strategic plan and the other the recalibrated forward model, subsequent movements will move away from the true task goal in the direction of the strategic target location. Strategic control has also been observed to disrupt performance on well-learned tasks. ${ }^{2}$ In these cases, the inference has been that the participant invokes control operations that, while useful for supporting rapid learning, may be slow in terms of on-line control.

For sequence learning, the distinction between fast multidimensional and slow unidimensional associative processes has provided a computational account of some fundamental differences between multiple learning systems. This hypothesis has not been applied to the studies of motor adaptation but there is some initial evidence for such a distinction. Divided attention manipulations can selectively disrupt the early phase of 
visuomotor adaptation, ${ }^{17}$ consistent with the hypothesis that a fast, cognitively demanding process can facilitate early adaptation. Similarly, divided attention can improve performance on well-learned skills (such as kicking a soccer ball), even though such manipulations hamper early learning. ${ }^{2}$

\section{OFF-LINE PROCESSING AND CONSOLIDATION}

Multiple learning mechanisms that operate at different timescales can provide a balance between flexibility and stability. Fast mechanisms rapidly develop new representations when confronted with a novel environment or task. However, they require greater attentional resources and processing time. Slower operating mechanisms can decrease cognitive load while ensuring that learned skills are retained in the face of novel learning. Indeed, a hallmark of motor skills is their robustness over long periods of time; we quickly become comfortable again on skis after a break of several months or even several years. Skills that are retained over extended intervals, even in the face of interference, are deemed 'consolidated.' Recent studies have focused on the fact that at least some aspects of consolidation occur 'off-line', that is, when the person is not actively practicing the skill.

Certain forms of learning require that participants sleep to obtain off-line benefits, whereas other forms are less restrictive, consolidating during any passage of time. The types of representations supported by fast processes-for example, those that are facilitated by awareness - seem especially prone to sleep-dependent consolidation (SDC). Again, awareness is not likely to be the key factor here. SDC during sequence learning is also observed when learning requires the integration of multidimensional cues, or contextual effects, even in the absence of awareness. ${ }^{18}$ The consolidation literature also points to the operation of multiple learning processes, with an interesting debate again emerging on whether these systems operate in parallel or in a competitive manner. For example, transcranial magnetic stimulation over primary motor cortex has been found to selectively disrupt performance measures correlated with slow learning processes. ${ }^{19}$ One particular intriguing study suggests that explicit learning may inhibit consolidation during wakefulness of sequence learning. Degrading explicit memory actually increased off-line reaction time (RT) gains that occurred during wakefulness. ${ }^{20}$

Consolidation processes during adaptation tasks have been more difficult to study, in large part, because once the experimental session ends, the person continues to move in a normal (nonperturbed) environment. Consolidation is frequently evident as savings in relearning; when the task is reintroduced after an extended break, performance improves faster than during initial acquisition. Consolidation is also observed in resistance to interference. For example, rotations learned with the wrist were robust to interference from a counter-rotation learned with arm movements when the adaptation tasks were sufficiently separated in time. Interestingly, when adaptation is followed by an extended period of performance in a nonperturbed environment (i.e., washout), resistance to interference is especially pronounced when testing is performed on the next day, even with the same effector (i.e., after sleep). In contrast, overtraining appears to produce similar resistance effects after either a 5-min break or a full-day break. ${ }^{21}$ Washout after fewer trials may be helpful in protecting more abstract representations that derive from fast learning processes, while overlearning 
may shift performance to more task/effector-specific representations that emerge from slower learning processes. Clearly, we must be cautious in testing such theories with respect to adaptation, but as with SRT learning, we may find a coherent multiple-system account of both learning dynamics and off-line consolidation.

As noted above, one important issue to keep in mind is whether the relationship between multiple learning systems is best thought of in terms of independent, parallel systems or as competitive subsystems. Some neuroimaging studies suggest that it may be more appropriate to view these systems as operating in a competitive manner rather than as parallel systems. ${ }^{1}$ However, this may be a function of the particular tasks used in these studies. In most conditions, multiple learning mechanisms converge toward a common solution. There are clear demonstrations of response-specific and context-specific facilitation being learned in parallel. $^{22}$

\section{CONCLUSIONS}

The skill acquisition literature is consistent with the notion that multiple, distinct systems support motor learning (Table 1). Ultimately, the most precise characterization of these will be in neural terms. For example, the various factors leading to sleep-dependent or independent consolidation may seem confusing in behavioral terms. In marked contrast, it has been proposed that SDC is limited to hippocampal-dependent learning. ${ }^{18}$ While this hypothesis may turn out to be incorrect, it provides an explicit computational account with strong points of connection to the memory literature. Rather than focus on task-based descriptions, an articulation of computational principles is likely to prove fruitful for understanding the mechanisms of motor learning as well as provide links to more general models of learning. In this review, we have noted the role of more general cognitive processes that influence skill acquisition. Conversely, many of the mechanisms and learning phenomena described in the recent literature on motor learning have a long history of application beyond motor skills. Chunking provides a method for the development of new objects in memory. Hierarchical organization imposes an organization on these objects and emphasizes how learning modifies associations within and between levels.

Only a portion of our skill learning capacities is accessible to consciousness, and these are likely to be the most flexible and 'high level.' Such systems likely require resources, in terms of time and attention to form associations that span complex contingency structures, provide the flexibility required for transfer and generalization, and learn from a limited number of experiences. Equally clearly, we seem to be endowed with implicit learning mechanisms that can readily learn task structures and contingencies. The time course and manner of training may have a marked effect on the relative roles for the various systems for skill learning, and these differences can have large consequences on immediate and consolidated performance.

At present, there is a broad experimental support for the architecture of the systems contributing to sequence learning. With respect to adaptation, only a handful of experiments have taken a multisystem approach, and our conclusions are more tentative. The common structure of adaptation and sequence learning is likely due to shared computational 
requirements, and perhaps even similar or shared neural systems that support these computations. On the other hand, adaptation may fail to share some of the features we have described for sequence learning-for example, a fast process that is penetrable to consciousness may be relevant for sequence learning and not for adaptation. The evaluation of these features across different aspects of skill learning provides us with an opportunity to create a coherent theory of motor function and learning.

\section{REFERENCES}

1. Poldrack RA, Clark J, Pare-Blagoev EJ, Shohamy D, Creso MJ, et al. Interactive memory systems in the human brain. Nature. 2001; 414:546-550. [PubMed: 11734855]

2. Beilock SL, Carr TH, MacMahon C, Starkes JL. When paying attention becomes counterproductive: impact of divided versus skill-focused attention on novice and experienced performance of sensorimotor skills. J Exp Psychol Appl. 2002; 8:6-16. [PubMed: 12009178]

3. Wulf $\mathrm{G}$, Weigelt $\mathrm{C}$. Instructions about physical principles in learning a complex motor skill: to tell or not to tell. Res Q Exerc Sport. 1997; 68:362-367. [PubMed: 9421849]

4. Seibel R. Discrimination reaction time for 1023-alternative task. J Exp Psychol. 1963; 66:215-226. [PubMed: 14047761]

5. Newell, A.; Rosenbloom, PS. Mechanisms of skill acquisition and the law of practice. In: Anderson, JR., editor. Cognitive Skills and Their Acquisition. Carnegie-Mellon University: Lawrence Erlbaum Associates, Inc.; 1981. p. 1-55.

6. Mackay DG. The problems of flexibility, fluency, and speed-accuracy trade-off in skilled behavior. Psychol Rev. 1982; 89:483-506.

7. Nissen MJ, Bullemer P. Attentional requirements of learning: evidence from performance measures. Cogn Psychol. 1987; 19:1-32.

8. Cohen A, Ivry RI, Keele SW. Attention and structure in sequence learning. J Exp Psychol Learn Mem Cogn. 1990; 16:17-30.

9. Keele SW, Jennings P, Jones S, Caulton D, Cohen A. On the modularity of sequence representation. J Mot Behav. 1995; 27:17-30.

10. Karni A, Meyer G, Jezzard P, Adams MM, Turner R, et al. Functional MRI evidence for adult motor cortex plasticity during motor skill learning. Nature. 1995; 377:155-158. [PubMed: 7675082]

11. Curran T, Keele SW. Attentional and nonattentional forms of sequence learning. J Exp Psychol Learn Mem Cogn. 1993; 19:189-202.

12. Keele SW, Ivry R, Mayr U, Hazeltine E, Heuer H. The cognitive and neural architecture of sequence representation. Psychol Rev. 2003; 110:316-339. [PubMed: 12747526]

13. Konkel A, Warren DE, Duff MC, Tranel DN, Cohen NJ. Hippocampal amnesia impairs all manner of relational memory. Front Hum Neurosci. 2008; 2:15. [PubMed: 18989388]

14. Schendan HE, Searl MM, Melrose RJ, Stern CE. An fMRI study of the role of the medial temporal lobe in implicit and explicit sequence learning. Neuron. 2003; 37:1013-1025. [PubMed: 12670429]

15. Smith MA, Ghazizadeh A, Shadmehr R. Interacting adaptive processes with different timescales underlie short-term motor learning. PLoS Biol. 2006; 4:e179. [PubMed: 16700627]

16. Mazzoni P, Krakauer JW. An implicit plan overrides an explicit strategy during visuomotor adaptation. J Neurosci. 2006; 26:3642-3645. [PubMed: 16597717]

17. Eversheim U, Bock O. Evidence for processing stages in skill acquisition: a dual-task study. Learn Mem. 2001; 8:183-189. [PubMed: 11533221]

18. Spencer RM, Sunm M, Ivry RB. Sleep-dependent consolidation of contextual learning. Curr Biol. 2006; 16:1001-1005. [PubMed: 16713957]

19. Hadipour-Niktarash A, Lee CK, Desmond JE, Shadmehr R. Impairment of retention but not acquisition of a visuomotor skill through time-dependent disruption of primary motor cortex. $\mathrm{J}$ Neurosci. 2007; 27:13413-13419. [PubMed: 18057199]

Wiley Interdiscip Rev Cogn Sci. Author manuscript; available in PMC 2015 March 02. 
20. Brown RM, Robertson EM. Inducing motor skill improvements with a declarative task. Nat Neurosci. 2007; 10:148-149. [PubMed: 17237776]

21. Krakauer JW, Ghez C, Ghilardi MF. Adaptation to visuomotor transformations: consolidation, interference, and forgetting. J Neurosci. 2005; 25:473-478. [PubMed: 15647491]

22. Race EA, Shanker S, Wagner AD. Neural priming in human frontal cortex: multiple forms of learning reduce demands on the prefrontal executive system. J Cogn Neurosci. 2008; 21:17661781. [PubMed: 18823245]

\section{FURTHER READING}

23. Hikosaka O, Nakamura K, Sakai K, Nakahara H. Central mechanisms of motor skill learning. Curr Opin Neurobiol. 2002; 12:217-222. [PubMed: 12015240]

24. Krakauer JW. Motor learning and consolidation: the case of visuomotor rotation. Adv Exp Med Biol. 2009; 629:405-421. [PubMed: 19227512]

25. Robertson EM. From creation to consolidation: a novel framework for memory processing. PLoS Biol. 2009; 7:e1000019.

26. Walker MP, Stickgold R. Sleep-dependent learning and memory consolidation. Neuron. 2004; 44:121-133. [PubMed: 15450165] 

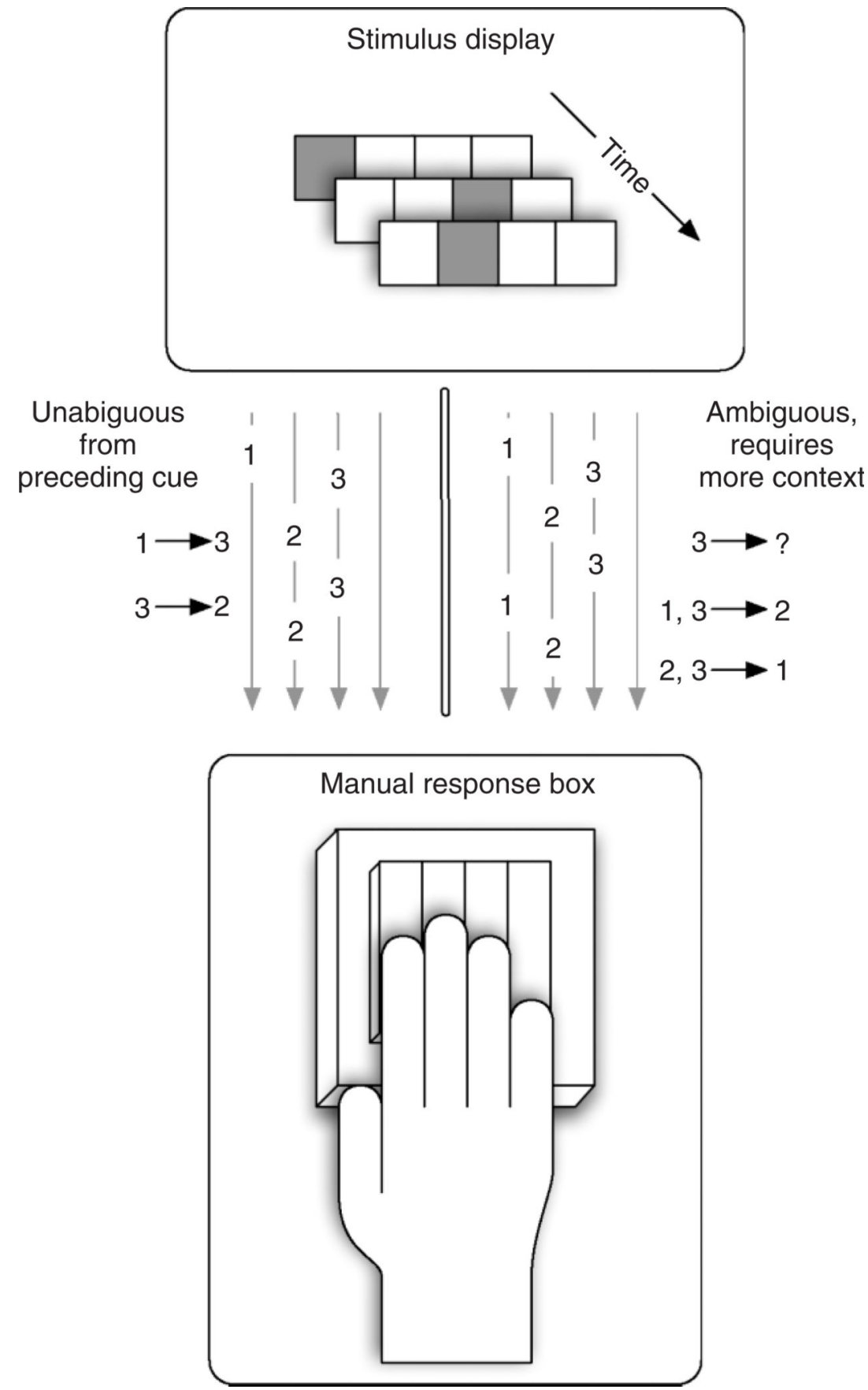

Figure 1.

The serial reaction time task. Stimuli corresponding to one of a discrete number of actions such as manual button presses are presented one at a time. With practice, participants are faster to respond when the stimuli follow a repeating sequence compared to when selected at random. The three trials here correspond to the first three elements of either the sequence on the left or right. Unambiguous contingencies (i.e., ' 3 ' always follows ' 1 ') are learned even when attention is divided by a concurrent secondary task. The individual elements are 
ambiguous in the sequence on the right. The context or higher order associations can be used to predict the next action. Such sequences are difficult to learn when attention is distracted. 
(a)

(c) Rotation + Strategy

(b)
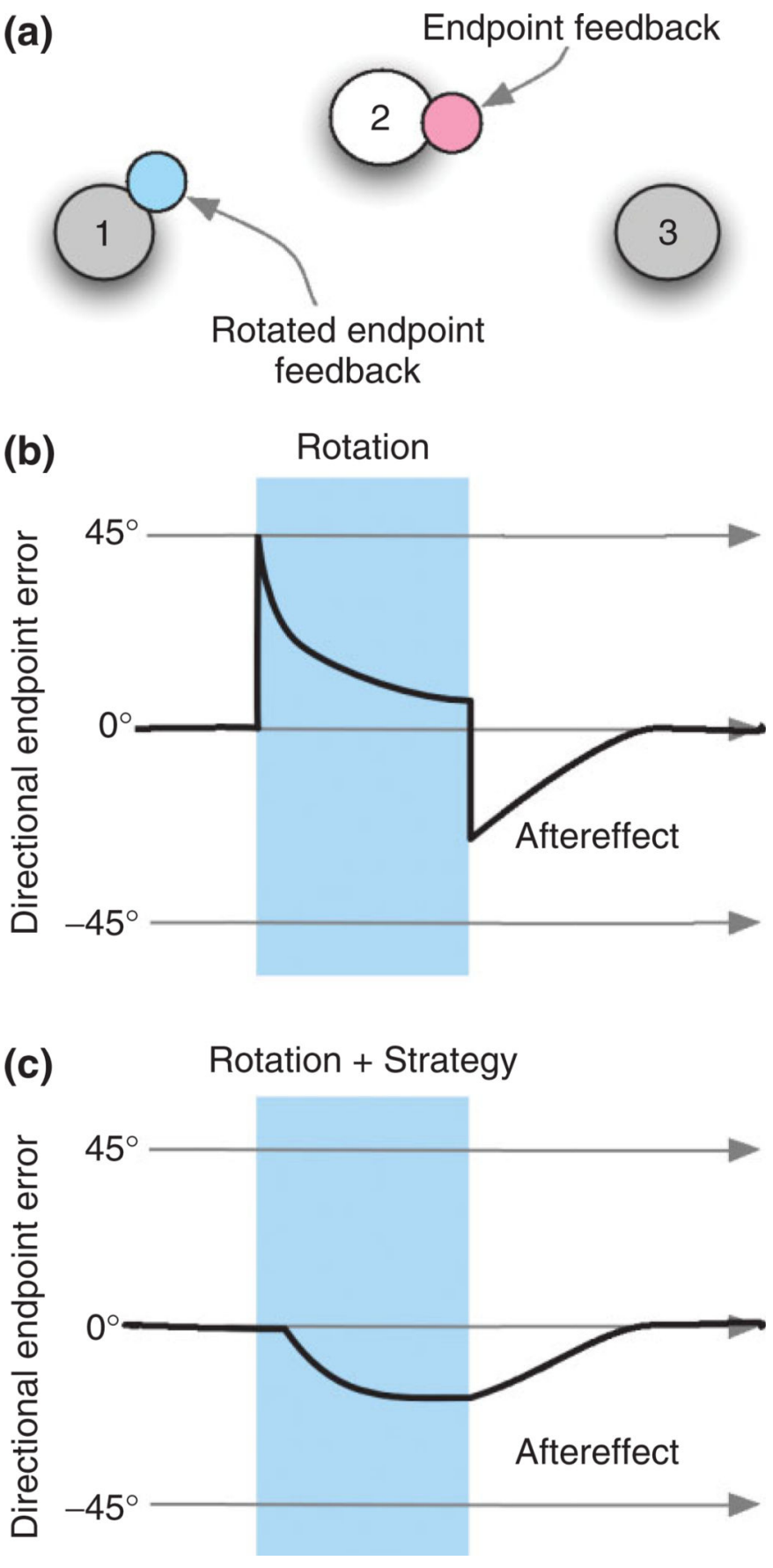

Figure 2.

Visuomotor adaptation. (a) The participant is instructed to reach to the illuminated target (target 2). The hand is not visible, and feedback for the movement is indicated by a cursor. Successful movements are those that result in the cursor arriving at the correct target. With the introduction of a $45^{\circ}$ counterclockwise rotation, previously correct movements to target 2 will result in the cursor arriving near target 1. (b) After a series of trials, a visual rotation is introduced by rotating the cursor feedback $45^{\circ}$ in the counterclockwise direction. Participants gradually recalibrate their movement such that the movement heading is rotated in the

Wiley Interdiscip Rev Cogn Sci. Author manuscript; available in PMC 2015 March 02. 
clockwise direction. When the rotation is turned off, an aftereffect is observed with error in the opposite direction. This washes out over subsequent trials. (c) Minimal error is observed on the initial learning trials when participants are instructed to use a clockwise compensation strategy that offsets the rotation [i.e., aiming at target 3 in panel(a)]. But even in the presence of this strategic correction, movements are again recalibrated in the clockwise direction, which degrades performance over time. When the rotation is removed and participants are told to cease using the strategy, a significant aftereffect is observed. Implicit recalibration occurs even when the strategy results in initially 'correct' movements. 


\section{TABLE 1}

\section{Characteristics of Multiple Systems for Motor Learning}

\begin{tabular}{ll}
\hline 'Fast' system(s) & 'Slow' system(s) \\
\hline Large amount of learning per trial that saturates quickly (high gain) & Small, incremental amount of learning per trial (low gain) \\
Requires extra time, cognitive resources for processing & Learns automatically without effort \\
Required for contextual learning & Unimodal or modular learning \\
Accessible to awareness and conscious intention & Impenetrable to awareness, operates independent of conscious strategies \\
Consolidation processes are enhanced during sleep & Consolidates off-line with the simple passage of time \\
Ready transfer to related tasks & Effector-specific and inflexible \\
\hline
\end{tabular}

Wiley Interdiscip Rev Cogn Sci. Author manuscript; available in PMC 2015 March 02. 